PROCEEDINGS OF THE

AMERICAN MATHEMATICAL SOCIETY

Volume 124, Number 4, April 1996

\title{
ON A GENERALISATION OF SELF-INJECTIVE VON NEUMANN REGULAR RINGS
}

\author{
GEORGE IVANOV
}

(Communicated by Ken Goodearl)

\begin{abstract}
Apart from von Neumann regular rings, rings with infinite identities have not been studied in any detail. We take a first step in that direction by obtaining structure theorems for a class of self-injective rings with infinite identities. These extend the main structure theorems for self-injective von Neumann regular rings.
\end{abstract}

A ring's identity element may be infinitely decomposable in that it cannot be expressed as the sum of (a finite number of) primitive idempotents. For brevity we will call such identity elements infinite and the others finite. This article is the result of an attempt to understand (a little of) the structure of rings with infinite identity elements. Except for von Neumann regular rings such rings have received little attention from algebraists. Von Neumann regular rings with finite identity elements are the semisimple Artinian rings (and are injective). It appears that in the class of rings with infinite identities the self-injective von Neumann regular rings play the same role - as the simplest, most basic examples.

Therefore our point of departure is to study a class of rings which includes the injective von Neumann regular rings but is sufficiently larger to be interesting. We choose the rings whose finitely generated (left) ideals are quasi-injective and call these rings (left) $f Q$-rings. They are a generalisation of $Q$-rings which have been of some interest in their own right (see e.g [I1], [I2] or [J] (and their references) the last paper has a survey of results in the area).

In $\S 1$ we show that $f Q$-rings with finite identities are $Q$-rings and therefore their structure is known completely (except for the rather uninteresting local case). The general case when the ring has infinite identity appears to be very difficult. To simplify the task we introduce two different concepts (dense primitive idempotents and idempotent nonsingular) both of which are intermediate between finite identity and arbitrary infinite identity but in different "directions". In $\S 1$ we show that the main structure theorem on injective von Neumann regular rings (the decomposition into Types) can be extended to $f Q$-rings which are idempotent nonsingular; and in $\S 2$ we determine the structure of indecomposable idempotent nonsingular $f Q$-rings with dense primitive idempotents and represent them as rings of matrices.

Throughout this paper all rings are unital, and all ideals, properties, etc., which are one-sided are left ideals, properties, etc. Right or two-sided ideals, properties,

Received by the editors June 7, 1994 and, in revised form, October 18, 1994

1991 Mathematics Subject Classification. Primary 16D50, 16D70; Secondary 16E60.

Honorary Associate at Macquarie University. 
etc., will specifically be so designated. The letters $e, f$ with or without superscripts or subscripts will always stand for idempotents. Idempotent will mean nontrivial idempotent (i.e. $\neq 0$ or 1$)$. The injective hull of a module $M$ will be denoted by $E(M)$. In the interest of brevity we will from now on refer to von Neumann regular rings as simply von Neumann rings. For results on von Neumann rings the reader is referred to Goodearl's book $[\mathrm{G}]$ which also contains basic information about quasi-injective modules.

\section{$\S 1$. IDEMPOTENT NONSINGULAR $f Q$-RINGS}

Definition. A ring's identity (element) is finite if it is a sum of primitive orthogonal idempotents, otherwise it is infinite. A set of idempotents is (left) dense in a ring if it generates an essential (left) ideal of the ring.

Clearly a ring has finite identity if and only if it is a (module) direct sum of indecomposable ideals. A ring with finite identity obviously has dense primitive idempotents. The structure of rings with identities is completely determined by their indecomposable ring summands. This is not true for rings with infinite identities and in fact such rings need not even have indecomposable summands.

In this section we introduce a concept of Types which is a natural extension of von Neumann's defintion but which is applicable to much larger classes of rings. We show that the main structure theory on injective von Neumann rings (the decomposition into Types) can be extended to a large class of $f Q$-rings under this new defintion. The example at the end of the paper shows that this class (the idempotent nonsingular rings) is the largest possible.

1.1 Lemma. An indecomposable nonlocal $f Q$-ring with primitive idempotents has nonzero socle. The image of any homomorphism between a principal indecomposable ideal and a disjoint ideal is simple.

Proof. Let $R$ be such a ring and let $e \in R$ be a primitive idempotent. Then either $e R(1-e) \neq 0$ or $(1-e) R e \neq 0$. First assume the latter and let $a \in(1-e) R e$ be nonzero. Then $R a$ is essential in $R e$, since $R e$ is an indecomposable injective, and $L=R a \oplus R(1-e)$ is quasi-injective and therefore invariant under endomorphisms of $R e \oplus R(1-e)$. That is, $(1-e) R e \subseteq R a$. As $a$ is arbitrary, this implies that $R a$ is simple and so $R$ has nonzero socle.

Now consider the case $e R(1-e) \neq 0$ and let $a \in e R(1-e)$ be any nonzero element. If $R f$ is the injective hull of $R a$, then $R a \oplus R e$ is quasi-injective and essential in $R f \oplus R e$. It follows, as above, that $e R f \subseteq R a$. Since $e R e$ is local ([F1], Proposition 5.8) $R a$ is indecomposable and so $R f$ is indecomposable. Hence $f$ is a primitive idempotent and so $R a$ is the unique subideal of $R f$ by the preceding paragraph.

1.2 Corollary. An indecomposable nonlocal $f Q$-ring with dense primitive idempotents has essential socle.

Proof. Let $e$ be a primitive idempotent in such a ring $R$. If $R e$ is simple, then the injective hull of all its images in $R$ is a ring summand of $R$ and therefore must be $R$. So we can assume that $R e$ is not simple. If $(1-e) R e \neq 0$, then, by Lemma $1.1, R e$ contains a simple ideal which must be essential in $R e$ since $R e$ is indecomposable and injective. So we need only consider the case $(1-e) R e=0$. Then $e R(1-e) \neq 0$ 
since $R$ is indecomposable and therefore the $R$-subideals of $R e$ and the $e R e$-ideals of $e R e$ coincide. If $L$ is a cyclic ideal contained in the radical $J$ of $e R e$, then since $e R e$ is local, there is a nonzero homomorphism from $L$ to $R(1-e)$. This homomorphism can be extended to a homomorphism $R e \rightarrow R(1-e)$ since $R(1-e)$ is injective. But by Lemma 1.1 every homomorphism $R e \rightarrow R(1-e)$ kills $J$ - a contradiction. Therefore $(1-e) R e \neq 0$ and $R e$ has nonzero socle.

1.3 Corollary. An indecomposable nonlocal fQ-ring with finite identity is a $Q$ ring.

Proof. If $e$ is a primitive idempotent in such a ring $R$, then $R e$ has essential socle $S$ (by Corollary 1.2) which must be simple and $e R e$ is local with radical $J$ consisting of zero divisors (Theorem 1.22 of [G]). As every endomorphic image of Re must contain $S$, this implies that $J=0$. Hence by Lemma 1.1, Re has at most one submodule. Therefore $R$ is a sum of Artinian ideals and so is itself Artinian. That is, $R$ is a $Q$-ring.

The structure of $Q$-rings with finite identities is determined in [I1] (except for the local case) so from now on we will only consider rings with infinite identities. Thus all our proofs will assume it and the interested reader is referred to [I1] for proofs of the finite identity case. But in the interest of completeness the statements of our results will be given in full generality.

1.4 Lemma. A nonsingular $f Q$-ring is a von Neumann regular ring.

Proof. If $R$ is an $f Q$-ring, then $R \cong \operatorname{End}(R)$ and so $R / J(R)$ is a von Neumann ring. But $J(R)$ is also the singular ideal of $R$ so if $R$ is nonsingular it is a von Neumann ring ([G], Theorem 1.22).

1.5 Lemma. Let $e, f$ be orthogonal idempotents in an $f Q$-ring $R$ and let $T$ be a subideal in Re. If $T$ has a nonzero image $S$ in $R f$, then $T$ contains an idempotent. If $S$ is cyclic, then it is the image of a subideal of $T$ generated by an idempotent.

Proof. Let $\phi: T \rightarrow S$ be an epimorphism. We may assume that $S$ is cyclic. If $S$ contains an idempotent $f^{\prime}$, then since $R f^{\prime}$ is projective, the pre-image of $R f^{\prime}$ under $\phi$ splits and therefore $T$ contains a copy of $R f^{\prime}$. But $R f^{\prime}$ is injective so this copy in $T$ is generated by an idempotent. So we can assume that $S$ contains no idempotents. Let $R e^{\prime}=E(T)$. Since $S$ is cyclic, $R e^{\prime} \oplus S$ is quasi-injective and so $\phi$ extends to a homomorphism $\Phi: R e^{\prime} \rightarrow S$. But then $T \Phi=S$ and $R e^{\prime} \Phi=S$ and it follows that $T=R e^{\prime}$. For if $T$ is a proper subideal of $R e^{\prime}$, then, since $k e r \Phi \subseteq T$, $T / \operatorname{ker} \Phi$ is a proper subideal of $\operatorname{Re} e^{\prime} \operatorname{Ker} \Phi=S$ - a contradiction. Hence $T=\operatorname{Re}^{\prime}$ and so $T$ contains an idempotent.

1.6 Lemma. Let $e, f$ be orthogonal idempotents in an $f Q$-ring $R$. If $R e \cong R f$, then every cyclic ideal in Re is injective.

Proof. Let $R a \subseteq R f$. Then $R a \oplus R e$ is quasi-injective and so $e R f_{1} \subseteq R a$ where $R f_{1}=E(R a)$. But $R e$ contains an isomorphic copy of $R f_{1}$, which must be a summand, and so there is an epimorphism $R e \rightarrow R f_{1}$. Therefore $e R f_{1}$ generates $R f_{1}$ and so $R a=R f_{1}$. Hence $R a$ is injective. 
1.7 Lemma. Let $e_{1}, e_{2}, e_{3}$ be orthogonal idempotents in an $f Q$-ring $R$. If $\alpha$ : $\operatorname{Re}_{1} \rightarrow \operatorname{Re}_{2}$ and $\beta: \operatorname{Re}_{2} \rightarrow \operatorname{Re}_{3}$ and the image of $\alpha$ does not contain injective ideals, then $\alpha \beta=0$.

Proof. $\operatorname{Re}_{1} \alpha$ does not contain an idempotent as it contains no injective ideals. Therefore, by Lemma 1.5, $R e_{1} \alpha$ cannot have an image in $R e_{3}$. Hence $\alpha \beta=0$.

Definition. An idempotent $e$ in an $f Q$-ring $R$ is abelian if all idempotents in the ring $e R e$ are central (in eRe). An $f Q$-ring is of Type I if it has dense abelian idempotents.

The standard definition of Type I for self-injective von Neumann rings (see [G]) is that such a ring is of Type I if it contains an abelian idempotent which is not orthogonal to any central idempotent (except zero).

1.8 Lemma. An injective von Neumann regular ring is of Type I, in our sense, if and only if it is of Type I in von Neumann's sense.

Proof. Let $R$ be of Type I in our sense. Then the two sided ideal generated by the abelian idempotents is essential as a left ideal and so $R$ is of Type I in von Neumann's sense by Theorem 10.4 of [G]. Now assume that $R$ is of Type I in von Neumann's sense. Then every ideal contains an abelian idempotent (by Theorem 10.4 of [G]) and so $R$ has an essential ideal generated by abelian idempotents.

Definition. A ring is (left) idempotent nonsingular if none of its elements is annihilated (on the left) by an essential (left) ideal generated by idempotents.

It is clear that nonsingular rings and rings with finite identities are idempotent nonsingular.

1.9 Theorem. An idempotent nonsingular fQ-ring is a direct sum of a ring with dense primitive idempotents and a ring with no primitive idempotents.

Proof. Let $R e$ be the injective hull of the ideal $P$ generated by the primitive idempotents in an $f Q$-ring $R$. By Lemma 1.1 any image $L$ in $R(1-e)$ of $R f$, for a primitive idempotent $f$, is simple so its injective hull is generated by a primitive idempotent. But $R(1-e)$ has no primitive idempotents so any homomorphism from Re to $R(1-e)$ must kill $P$. This contradicts the assumption that $R$ is idempotent nonsingular. Hence $e R(1-e)=0$.

Conversely, if $f \in R e$ is a primitive idempotent, then any homomorphism $\alpha$ : $R(1-e) \rightarrow R f$ has a simple image (by Lemma 1.1). Then the kernel $K$ of $\alpha$ must be essential since $R(1-e)$ has no simples. We now show that the ideal $I$ generated by the idempotents in $K$ is essential in $K$. Let $x \in K$ be any element and consider $R f_{1}=E(R x)$. If $R f_{1}$ is in $I$ there is nothing to prove. If $R f_{1}$ is not in $K$, then $R f_{1} /\left(R f_{1} \cap K\right)$ is simple. But $f_{1}$ is not primitive so it is a sum of orthogonal idempotents $g_{1}, g_{2}$ and one of these is in $K$, say $g_{1}$. But $R x \cap R g_{1} \neq 0$ so $I \cap R x \neq 0$ as required. Thus we have shown that there can be no homomorphism $R(1-e) \rightarrow R f$ for any primitive idempotent $f \in R e$ and so it follows that $(1-e) R e=0$. Therefore $R$ decomposes as claimed.

1.10 Theorem. An idempotent nonsingular $f Q$-ring is a direct sum of a ring with essential singular ideal and a von Neumann regular ring.

Proof. Let $Z(R)$ be the singular ideal of an idempotent nonsingular $f Q$-ring $R$ and let $R e=E(Z(R))$. As a homomorphic image of a singular module is singular, 
there are no images in $R(1-e)$ of $R e$. That is $e R(1-e)=0$. As $R(1-e)$ is nonsingular, its endomorphism ring $(1-e) R(1-e)$ is a von Neumann ring. Assume that $(1-e) R e \neq 0$ and let $x \in(1-e) R e$ be nonzero. The annihilator $\alpha(x)$ of $x$ in $R(1-e)=(1-e) R(1-e)$ is an essential subideal and must contain an essential subideal generated by idempotents since $(1-e) R(1-e)$ is a von Neumann ring. This contradicts the assumption that $R$ is idempotent nonsingular.

1.11 Corollary. If an indecomposable idempotent nonsingular $f Q$-ring $R$ has orthogonal idempotents $e, f$ such that $R e \cong R f$, then $R$ is a von Neumann regular ring.

Proof. By Lemma 1.6 every cyclic ideal in $R e$ is injective and thus is generated by an idempotent. Hence $R e$ is nonsingular. As $R$ is indecomposable, Theorem 1.10 implies that all of $R$ is nonsingular and thus a von Neumann ring.

We now prove the first of the main decomposition theorems which show that the theory of types which applies to injective von Neumann rings can be extended to idempotent nonsingular $f Q$-rings.

1.12 Theorem. An idempotent nonsingular $f Q$-ring is the direct sum of a ring of Type $I$ and $a$ ring with no abelian idempotents.

Proof. Let $A$ be the set of abelian idempotents in an idempotent nonsingular $f Q$ $\operatorname{ring} R$ and let $R e=E(R A)$. We want to show that $e R(1-e)=0=(1-e) R e$. First consider $e R(1-e)$ and assume that $e R(1-e) \neq 0$. Since $R$ is idempotent nonsingular, there is an $f \in A$ such that $f R(1-e) \neq 0$. Pick any nonzero $a \in$ $f R(1-e)$ and let $R e^{\prime}=E(R a)$. Then $e^{\prime}$ is infinite, for otherwise it would be a sum of abelian idempotents and would thus be in $R e$. So there are orthogonal idempotents $e_{1}, e_{2} \in R e^{\prime}$ such that $e_{1} R e_{2} \neq 0$. Let $c \in e_{1} R e_{2}$ be nonzero. As $R c \cap R a \neq 0$, there is an $R b \subseteq R c$ which is the image of a $K \subseteq R f$. By Lemma 1.5, $R b$ is an image of $R f^{\prime} \subseteq R f$ and so $f^{\prime} R(1-e) \neq 0$. But $R b$ is also an image of $R e_{1}^{\prime}$ for an $e_{1}^{\prime} \in R e_{1}$ and therefore $R f^{\prime}$ and $R e_{1}^{\prime}$ have isomorphic summands. As $f^{\prime}$ is abelian, any summand of $R f^{\prime}$ is generated by an abelian idempotent so $R e_{1}^{\prime}$ contains an abelian idempotent - a contradiction to the fact that $e_{1}^{\prime} \in R(1-e)$. Therefore $e R(1-e)=0$.

We now show that $(1-e) R e=0$. Assume not and let $\alpha: R(1-e) \rightarrow R e$ be a nonzero homomorphism. We can regard $\alpha$ as an endomorphism of $R$. By Lemma 1.5, $\alpha$ kills every subideal of $R$ which has no idempotents so $\alpha$ kills $Z(R)$, the singular ideal of $R$. Therefore $\alpha$ can be considered to be a homomorphism from $\bar{R}=R / Z(R)$. By Theorem 19.27 of [F2], $\bar{R}$ is a self-injective von Neumann ring. As $R$ is idempotent nonsingular, $R \alpha=\bar{R} \alpha$ is a nonsingular $\bar{R}$-module. Therefore $\overline{\operatorname{ker} \alpha}$ is a summand of $\overline{R(1-e)}$ and so $R(1-e) \alpha=\overline{R(1-e)} \alpha$ is isomorphic to a summand of $\overline{R(1-e)}$. As idempotents lift from $\bar{R}$ to $R$, there is an idempotent $f \in R(1-e)$ such that $\overline{\operatorname{ker} \alpha}=\overline{R(1-e-f)}$. Then $\left.\alpha\right|_{\overline{R f}}$ is an isomorphism. Let $f_{1} \in R f$ be an idempotent and $f_{2}=f-f_{1}$. Then $f_{i} R f_{j}=0$, for $i \neq j$, as otherwise $R f_{j}$ contains a homomorphic image of $\overline{R f_{i}} \cong R f_{i} \alpha$, so there would be a homomorphism from a subideal of $R e$ to $R f_{j}$ - a contradiction. Hence the idempotents $f_{1}, f_{2}$ are central in $f R f$ which means that $f$ is abelian - a contradiction. Therefore $(1-e) R e=0$ and the required decomposition follows. 
Definition. A module is finitary or von Neumann finite if it cannot be expressed as a direct sum of two nonzero submodules, one of which is isomorphic to the module itself. An idempotent is finitary or von Neumann finite if the ideal generated by it is finitary. A module (idempotent) is infinitary or von Neumann infinite if it is not finitary. An $f Q$-ring is of Type II if it has dense finitary idempotents but has no abelian idempotents. An $f Q$-ring is of Type III if it contains no finitary idempotents.

1.13 Lemma. An injective von Neumann regular ring is of Type II in our sense if and only if it is of Type II in von Neumann's sense.

Proof. As for Lemma 1.8 using Theorem 10.5 of [G].

1.14 Theorem. An fQ-ring of Type III is a von Neumann regular ring of Type III.

Proof. Let $R$ be an $f Q$-ring of Type III and let $R=L_{1} \oplus L_{2}$ where $L_{1} \cong R$. Then $L_{1}=L_{11} \oplus L_{12}$ where $L_{11} \cong L_{1}$ and $L_{12} \cong L_{2}$. By Lemma 1.6 every cyclic subideal of $L_{2}$ is injective. Now let $a \in L_{1}$ be nonzero and assume that $R a$ contains no injective ideals. Let $R e=E(R a)$. Then since $R$ has no finitary idempotents, $R e=T_{1} \oplus T_{2}$, where $T_{1} \cong R e$. By the above argument every cyclic ideal in $T_{2}$ is injective. As $R a$ is essential in $R e, R a \cap T_{2} \neq 0$, which means that $R a$ contains injective ideals - a contradiction to our assumption. Therefore every cyclic ideal in $L_{1}$ is injective and so $R$ is a von Neumann ring.

We now prove the final theorem on decomposition into types.

1.15 Theorem. An idempotent nonsingular $f Q$-ring with no abelian idempotents is a direct sum of a ring of Type II and a ring of Type III.

Proof. Let $R$ be an idempotent nonsingular $f Q$-ring without abelian idempotents and let $X$ be the set of finitary idempotents in $R$. Let $R e=E(R X)$. We want to show that $e R(1-e)=(1-e) R e=0$.

Let $f \in R(1-e)$. Then $R f=R f_{1} \oplus R f_{2}$ where $R f \cong R f_{1}$. By Lemma 1.6 every cyclic subideal of $R f$ is generated by an idempotent and is injective. Hence if $0 \neq a \in e R(1-e)$, then the pre-image $P$ of $R a$ in $R e$ under any homomorphism contains a copy of $R a$. Now $R a$ is infinitary and all its cyclic subideals are injective and infinitary. Therefore $R X \cap P$ contains infinitary idempotents - a contradiction. Hence $e R(1-e)=0$.

Since $R(1-e)$ has no finitary idempotents, the ring $(1-e) R(1-e)$ is of Type III and is a von Neumann ring by Theorem 1.14. Let $x \in(1-e) R e$ and assume $x$ is nonzero. If the annihilator $\alpha(x)$ of $x$ in $R(1-e)$ is not essential, then there is a $c \in R(1-e)$ such that $R c \cap \alpha(x)=0$. Then $R c \cong R c x$. As $R c$ is generated by an infinitary idempotent, it is injective and so $R c x$ is generated by an infinitary idempotent - a contradiction to the definition of Re. Therefore $\alpha(x)$ must be essential in $R(1-e)$. But then $\alpha(x)$ contains an essential subideal generated by idempotents, since $R(1-e)=(1-e) R(1-e)$ is a von Neumann ring, which contradicts the assumption that $R$ is idempotent nonsingular. So $(1-e) R e=0$ as required.

\section{$\S 2$. $f Q$-RINGS With DENSE PRIMitive IDEMPotents}

Rings with dense primitive idempotents have indecomposable summands and in the case of $f Q$-rings these determine the structure of the rings. In this section we 
determine the structure of indecomposable idempotent nonsingular $f Q$-rings with dense primitive idempotents by representing them as rings of matrices. Example 2.6 shows that the idempotent nonsingular assumption is essential. Except for the statement of the main result (Theorem 2.3) we will only be concerned with rings which are not von Neumann rings and not local.

2.1 Lemma. Every minimal ideal in an idempotent nonsingular $f Q$-ring is the image of an ideal generated by a primitive idempotent.

Proof. Let $R$ be an idempotent nonsingular $f Q$-ring and let $M$ be a minimal ideal in $R$ with injective hull $R e$. If $e M \neq 0$ there is nothing to prove since $e$ must be a primitive idempotent. Therefore we need only consider the case when $M$ is an image of $R(1-e)$. Since $M$ is minimal, its annihilator $\alpha(M)$ in $R(1-e)$ is a maximal subideal. If $\alpha(M)$ is not essential in $R(1-e)$, then its complement is a minimal ideal (isomorphic to $M$ ) which is a summand of $R(1-e)$ and is therefore generated by a primitive idempotent as required. If $\alpha(M)$ is essential in $R(1-e)$, then it must contain an essential subideal generated by idempotents.

For let $x \in \alpha(M)$ be any element and let $R f$ be the injective hull of $R x$. If $R f \subseteq \alpha(M)$, then $R x$ intersects the subideal of $\alpha(M)$ generated by idempotents. So we can assume that $R f \subsetneq \alpha(M)$. Then $R f /(R f \cap \alpha(M))$ is simple and isomorphic to $M$. If $f$ is primitive, then there is nothing to prove so we assume that $f=f_{1}+f_{2}$ for orthogonal idempotents $f_{1}$ and $f_{2}$. One of $f_{1}, f_{2}$ must be in $\alpha(M)$, say $f_{1}$. As $R x$ is essential in $R f, R x \cap R f_{1} \neq 0$ and so $R x$ intersects the subideal of $\alpha(M)$ generated by idempotents, as required. But this means that $M$ is annihilated by an essential ideal generated by idempotents contrary to the assumption about $R$. $\square$

Definition. An idempotent chain is a sequence of primitive idempotents $\left\{e_{i} \mid i \in\right.$ $I\}$ such that $\operatorname{Soc}\left(R e_{i}\right)$ is an image of $R e_{i+1}$ for all $i \in I$ where $I$ is a segment in $\mathbb{Z}$ unbounded above.

2.2 Lemma. Let $R$ be an indecomposable idempotent nonsingular $f Q$-ring with dense primitive idempotents. If $R$ is not local and not a von Neumann regular ring, then it has a unique idempotent chain.

Proof. Let $e_{0} \in R$ be a primitive idempotent and assume that $R$ is not von Neumann and not local. Then one of $e_{0} R\left(1-e_{0}\right)$ and $\left(1-e_{0}\right) R e_{0}$ is nonzero. Assume the former. By Lemma 1.1 any image of $R e_{0}$ in $R\left(1-e_{0}\right)$ is simple. By Corollary 1.11 it is unique. Let $R e_{-1}$ be its injective hull. If $R e_{-1}$ has an image in $R\left(1-e_{-1}\right)$, then that image is simple. Denote its injective hull by $R e_{-2}$. In this manner we can construct a sequence of disjoint ideals $R e_{n}, n=-1,-2,-3, \ldots$, such that $R e_{n}$ has a nonzero image in $R e_{n-1}$. As we have assumed that $R$ is not a von Neumann ring, $R e_{0}$ is not simple (Lemma 1.6) and so it has a proper subideal which must be its socle (Lemma 1.1). By Lemma 2.1, $\operatorname{Soc}\left(\operatorname{Re}_{0}\right)$ is an image of an ideal generated by a primitive idempotent. By Corollary 1.11 this ideal is unique. Denote it by $R e_{1}$. The socle of $R e_{1}$ is an image of an ideal $R e_{2}$ generated by a primitive idempotent, and so on. In this manner we obtain a sequence of primitive idempotents $e_{n}$ for all integers $n$. This sequence $\mathcal{C}$ may be finite on the left, that is, $e_{k}=0$ for some negative integer $k$ in which case $e_{i}=0$ for all $i<k$. As our standing assumption is that $R$ does not have a finite identity, $\mathcal{C}$ will always be infinite on the right as we shall see below. 
Let $\mathcal{C}$ and $\mathcal{C}^{\prime}$ be two idempotent sequences in $R$ and assume that there is an idempotent $e$ which belongs to both of them. It follows that the sequences $\mathcal{C}$ and $\mathcal{C}^{\prime}$ coincide to the right of $e$ since $\operatorname{Soc}(R e)$ is the image of a unique $R f \subseteq R(1-e)$. As $R e$ has a unique image in $R(1-e), \mathcal{C}$ and $\mathcal{C}^{\prime}$ coincide to the left of $e$. That is, $\mathcal{C}=\mathcal{C}^{\prime}$. Therefore the idempotent chains partition the set of primitive idempotents in $R$.

We now show that there can only be one idempotent chain in $R$. Let $R f=$ $E(R \mathcal{C})$ and consider $R(1-f)$. Since $R$ is idempotent nonsingular and every $R e$, for $e \in \mathcal{C}$, has images only in $R \mathcal{C}$, there cannot be any homomorphisms from $R f$ to $R(1-f)$. That is, $f R(1-f)=0$. Since every simple ideal in $R \mathcal{C}$ can be an image only of an $R e$ for some $e \in \mathcal{C}$, there are no homomorphisms $R(1-f) \rightarrow R f$. Therefore $(1-f) R f=0$ and so $R f$ is generated by a central idempotent. Since $R$ is indecopmposable, this means that $R f=R$.

Now assume that an idempotent $e$ occurs twice in $\mathcal{C}$. Then $\mathcal{C}$ contains only a finite number of distinct idempotents and so $R$ must have finite identity. Therefore all the idempotents in $\mathcal{C}$ are distinct and $\mathcal{C}$ is infinite - at least to the right.

Definition. Let $D$ be a sfield and $V$ a null $D$-algebra one dimensional on both sides. If $\mathbb{N}$ and $\mathbb{Z}$ denote the sets of positive, respectively all, integers, then the rings $H(\mathbb{N}, D, V)$ and $H(\mathbb{Z}, D, V)$ are defined to be the following matrix rings.

$$
\begin{gathered}
H(\mathbb{N}, D, V)=\left(\begin{array}{llll}
D & & & \\
V & D & & \\
& V & D & \\
& & \ddots & \ddots
\end{array}\right) \quad \text { with } \mathbb{N} \text { rows and columns. } \\
H(\mathbb{Z}, D, V)=\left(\begin{array}{lllll}
\ddots & & & & \\
\ddots & D & & & \\
& V & D & & \\
& & V & D & \\
& & & \ddots & \ddots
\end{array}\right) \quad \text { with } \mathbb{Z} \text { rows and columns. }
\end{gathered}
$$

2.3 Theorem. Let $R$ be an indecomposable idempotent nonsingular $f Q$-ring with infinite identity and dense primitive idempotents. If $R$ is not a von Neumann regular ring, then it is isomorphic to one of the rings $H(\mathbb{N}, D, V), H(\mathbb{Z}, D, V)$ for some sfield $D$.

Proof. Assume that $R$ is not a von Neumann ring. By Lemma 2.2, $R$ has a unique idempotent chain $\mathcal{C}$ and $R$ is the injective hull of $I=R \mathcal{C}$. For any $r \in R$ and $e \in \mathcal{C}$ the ideal $R e r$ is an image of $R e$ and is therefore in $I$. That is, $I$ is a two sided ideal. Therefore any endomorphism of $R$ induces an endomorphism of $I$. Conversely, since $R$ is injective, every endomorphism of $I$ extends to an endomorphism of $R$. This extension is unique. For if two endomorphisms of $R$, obtained by right multiplication by $x$ and $y$, say, coincide on $I$, then $I(x-y)=0$. Since $R$ is idempotent nonsingular this is only possible if $x=y$. Therefore $R \cong \operatorname{End}(R) \cong$ $\operatorname{End}(I)$ and we obtain the required representation of $R$ from that of $\operatorname{End}(I)$.

Let $e \in \mathcal{C}$ be any idempotent. We know $R e$ has a unique subideal which is not an image of $R e$. Therefore $E n d(R e)$ is a sfield. The socle of $R e$ is quasi-injective 
so is invariant under $e R e$ and therefore endomorphims of $S o c(R e)$ lift uniquely to $\operatorname{End}(R e)$. That is, $S o c(R e)$ is a one dimensional right vector space over $e R e$. Since $\operatorname{Soc}(R e)$ is an image of $R e^{\prime}$, where $e^{\prime}$ is the successor of $e$ in $\mathcal{C}$, it is a one dimensional vector space over $e^{\prime} R e^{\prime}$. Therefore $e R e \cong e^{\prime} R e^{\prime}$ as sfields. Hence we can denote all the $e R e^{\prime} s$ by $D$ and all the bimodules $e^{\prime} R e$ by $V$. Since $e^{\prime \prime} R e^{\prime} \cdot e^{\prime} R e=0$, we can regard the $V^{\prime} s$ as null algebras.

Now $I=\bigoplus_{e \in \mathcal{C}} R e$, so $\operatorname{End}(I)$ can be represented as the ring of all $\mathbb{N} \times \mathbb{N}$ or $\mathbb{Z} \times \mathbb{Z}$ row finite matrices whose entries at the place $(i, j)$ are arbitrary elements of $\operatorname{Hom}\left(R e_{i}, R e_{j}\right)$. From Lemma 2.2 these are nonzero only if $i=j$ or $i=j+1$. Using the above identifications this gives that $R$ is isomorphic to one of the rings $H(\mathbb{N}, D, V), H(\mathbb{Z}, D, V)$ depending on the sequence type of $\mathcal{C}$.

2.4 Corollary. A nonlocal indecomposable idempotent nonsingular fQ-ring with infinite identity and dense primitive idempotents need not be right injective.

Proof. The ring $H=H(\mathbb{N}, D, V)$ is not right injective since $e H$, where $e$ is the matrix whose only nonzero entry is the identity of $D$ at the place $(1,1)$, is not injective. But $H$ is very close to being right injective since all the other principal indecomposable right ideals are injective.

Definition. Let $D$ be a sfield and $V$ a null $D$-algebra one dimensional on both sides. The ring of all row finite $\omega \times \omega$-matrices over $D$ is denoted by $M_{\omega}(D)$. For an integer $m \geq 2$, define $H(m, D, V)$ to be the following matrix ring.

$$
H(m, D, V)=\left(\begin{array}{ccccc}
D & & & & V \\
V & D & & & \\
& V & \ddots & & \\
& & \ddots & D & \\
& & & V & D
\end{array}\right) \quad \text { with } m \text { rows and columns. }
$$

It is well known that the only indecomposable self-injective von Neumann rings with dense primitive idempotents are the rings $M_{\omega}(D)([\mathrm{G}]$, Theorem 9.12). It is shown in [I1] that the rings $H(m, D, V)$ are the only indecomposable $Q$-rings with finite identities which are not von Neumann rings or local rings.

2.5 Corollary. A ring is an idempotent nonsingular $f Q$-ring with dense primitive idempotents if, and only if, it is a product of rings of the following types: local fQ-rings, $M_{\omega}(D), H(m, D, V), H(\mathbb{N}, D, V)$ and $H(\mathbb{Z}, D, V)$.

Proof. Every primitive idempotent is contained in an indecomposable (ring) summand. Each of these is isomorphic to one of the rings listed above. The product of these rings is their injective hull. Therefore the ring is isomorphic to the product of the subrings. A standard argument shows that a product of such rings is an $f Q$-ring.

2.6 Example. Let $A$ be a von Neumann ring, let $V$ be a singular simple $A$-module and let $D$ be the endomorphism sfield of $V$. Let $R$ be the matrix $\operatorname{ring}\left(\begin{array}{cc}D & 0 \\ V & A\end{array}\right)$. If $A$ is of Type III, then $R$ is not idempotent nonsingular, it cannot be decomposed into Types and it is not a direct sum of a nonsingular ring and a ring with essential singular ideal. It does not decompose into a ring with dense primitive idempotents 
and a ring with no primitive idempotents. This shows that the idempotent nonsingular assumptions cannot be dropped from Theorems 1.9, 1.10, 1.12 and 1.15. If $A=M_{\omega}(D)$, for infinite $\omega$, then $R$ has dense primitive idempotents but it does not satisfy Theorem 2.3.

\section{ACKNOWLEDGEMENT}

The author is indebted to the referee for his many helpful suggestions about improving the presentation of this paper; and to Safdar Raza Syed for pointing out an error in a proof.

\section{REFERENCES}

[F1] C. Faith, Lectures on Injective Modules and Quotient Rings, Lecture Notes in Mathematics 49 (1967), Springer-Verlag. MR 37:2791

[F2] _ Algebra II. Ring Theory, Springer-Verlag, New York, 1976. MR 55:383

[G] K.R. Goodearl, Von Neumann Regular Rings, Pitman, London, 1979. MR 80e:16011

[I1] G. Ivanov, Non-local rings whose ideals are quasi-injective, Bull. Aust. Math. Soc. 6 (1972), 45-52. MR 45:311

[I2] G. Ivanov, Rings with quasi-injective ideals, Bull. Aust. Math. Soc. 50 (1994), 197-204. CMP 95:02

[J] S. K. Jain, Rings whose cyclic modules have certain properties and the duals, Lecture Notes Series 25 (1976), Marcel Dekker, New York, 143-160.

Department of Mathematics, Macquarie University, Sydney, Australia 2109

E-mail address: ivanov@mpce.mq.edu.au 